\title{
Silver Nanoparticles: Biosynthesis Using an ATCC Reference Strain of Pseudomonas aeruginosa and Activity as Broad Spectrum Clinical Antibacterial Agents
}

\author{
Melisa A. Quinteros, ${ }^{1}$ Ivana M. Aiassa Martínez, ${ }^{2}$ Pablo R. Dalmasso, ${ }^{3}$ and Paulina L. Páez \\ ${ }^{1}$ IMBIV, CONICET, Departamento de Farmacia, Facultad de Ciencias Químicas, Universidad Nacional de Córdoba, \\ Ciudad Universitaria, 5000 Córdoba, Argentina \\ ${ }^{2}$ UNITEFA, CONICET, Departamento de Farmacia, Facultad de Ciencias Químicas, Universidad Nacional de Córdoba, \\ Ciudad Universitaria, 5000 Córdoba, Argentina \\ ${ }^{3}$ CITSE, CONICET, Universidad Nacional de Santiago del Estero, RN 9, Km 1125, 4206 Santiago del Estero, Argentina \\ Correspondence should be addressed to Pablo R. Dalmasso; p-dalmasso@hotmail.com
}

Received 1 December 2015; Revised 22 April 2016; Accepted 15 May 2016

Academic Editor: Vijaya Kumar Rangari

Copyright (C) 2016 Melisa A. Quinteros et al. This is an open access article distributed under the Creative Commons Attribution License, which permits unrestricted use, distribution, and reproduction in any medium, provided the original work is properly cited.

\begin{abstract}
Currently, the biosynthesis of silver-based nanomaterials attracts enormous attention owing to the documented antimicrobial properties of these ones. This study reports the extracellular biosynthesis of silver nanoparticles (Ag-NPs) using a Pseudomonas aeruginosa strain from a reference culture collection. A greenish culture supernatant of P. aeruginosa incubated at $37^{\circ} \mathrm{C}$ with a silver nitrate solution for $24 \mathrm{~h}$ changed to a yellowish brown color, indicating the formation of Ag-NPs, which was confirmed by UVvis spectroscopy, transmission electron microscopy, and X-ray diffraction. TEM analysis showed spherical and pseudospherical nanoparticles with a distributed size mainly between 25 and $45 \mathrm{~nm}$, and the XRD pattern revealed the crystalline nature of AgNPs. Also it provides an evaluation of the antimicrobial activity of the biosynthesized Ag-NPs against human pathogenic and opportunistic microorganisms, namely, Staphylococcus aureus, Staphylococcus epidermidis, Enterococcus faecalis, Proteus mirabilis, Acinetobacter baumannii, Escherichia coli, P. aeruginosa, and Klebsiella pneumonia. Ag-NPs were found to be bioactive at picomolar concentration levels showing bactericidal effects against both Gram-positive and Gram-negative bacterial strains. This work demonstrates the first helpful use of biosynthesized Ag-NPs as broad spectrum bactericidal agents for clinical strains of pathogenic multidrug-resistant bacteria such as methicillin-resistant $S$. aureus, A. baumannii, and E. coli. In addition, these Ag-NPs showed negligible cytotoxic effect in human neutrophils suggesting low toxicity to the host.
\end{abstract}

\section{Introduction}

The continuing appearance of antibiotic resistance in pathogenic and opportunistic microorganisms obliges the scientific community to constantly develop new drugs and drug targets. The costs of healthcare-associated infections are clearly high and increasing as the number of infections that are caused by multiple drug-resistant microorganisms increases [1]. More than 70\% of bacterial nosocomial infections are resistant to one or more of the antibiotics traditionally used to treat them, and people infected with drugresistant microorganisms usually spend more time in the hospital and require a treatment that uses two or three different antibiotics which is less effective, more toxic, and more expensive [2].

Even though the goal of many scientists is designing drugs acting via novel mechanisms of action, few new antibiotics have been introduced by the pharmaceutical industry in the last decade, and none of them have improved the activity against multidrug-resistant bacteria [3]. In the current scenario, nanotechnology offers opportunities to reexplore the biological properties of already known antimicrobial materials by manipulating their size to alter the effect [4].

Recently, the application of nanoparticles in various fields has expanded considerably. Nanoparticles possess unique 
physicochemical characteristics, such as a high ratio of surface area to mass, high reactivity, and sizes in the range of nanometers $\left(10^{-9} \mathrm{~m}\right)$. Nanoparticles have been successfully used in nanochemistry to enhance the immobilization and activity of catalysts, in sensors, in medical and pharmaceutical nanoengineering for delivery of therapeutic agents, and in the food industry to limit bacterial growth [5-8]. Due to nanoparticles which have also demonstrated antimicrobial activities, the development of novel applications in this field makes them an attractive alternative to antibiotics.

In recent years, there has been growing interest in the synthesis and study of silver nanoparticles (Ag-NPs), because silver has long been known for its antimicrobial properties and the Ag-NPs are considered as nontoxic and environmentally friendly antibacterial materials that may be linked to broad spectrum activity and far lower propensity to induce microbial resistance compared to antibiotics $[8,9]$. Currently, many methods have been reported for the synthesis of AgNPs by using chemical, physical, and biological routes [10]. The latter has emerged as a green alternative and it is highly advantageous for it is eco-friendly, cost-effective, and easily scaled up. The biosynthesis of Ag-NPs has great potential with natural reducing agents and/or stabilizing compounds from bacteria, fungi, yeasts, algae, or plants $[10,11]$.

In this work, we provide a simple and eco-friendly strategy for the green synthesis of Ag-NPs using the metalreducing culture supernatant of Pseudomonas aeruginosa ATCC 27853. UV-vis spectroscopy and transmission electron microscopy were used to characterize the Ag-NPs biosynthesized. While a similar strategy has been used previously by Kumar and Mamidyala [12], this work provides the first extracellular biosynthesis of Ag-NPs using a $P$. aeruginosa strain from a reference culture collection. Also we evaluated the in vitro antimicrobial efficacy of the Ag-NPs against representative Gram-positive and Gram-negative bacteria such as Staphylococcus aureus, Staphylococcus epidermidis, Enterococcus faecalis, Proteus mirabilis, Acinetobacter baumannii, Escherichia coli, P. aeruginosa, and Klebsiella pneumoniae. To the best of our knowledge, this is the first work reporting the helpful use of the biosynthesized Ag-NPs as bactericidal agents for clinical strains of multiresistant human pathogenic microorganisms, namely, methicillin-resistant S. aureus, A. baumannii, and $E$. coli. In addition, we are submitting the preliminary results of cell viability assays of biosynthesized Ag-NPs-treated human neutrophils.

\section{Materials and Methods}

2.1. Reagents. Tryptic soy broth (TSB) and Mueller Hinton broth (MHB) were obtained from BritaniaLab and prepared according to manufacturer's recommendations. Silver nitrate (>99\% purity) was purchased from Cicarelli, Argentina, and employed to prepare fresh silver solutions $(10 \mathrm{mM})$ in sterile distilled water for each experiment. Dextran from Leuconostoc mesenteroides (average molecular weight 78,000), FicollHypaque (Histopaque-1077), and Trypan blue solution were obtained from Sigma. Hank's balanced salt solution (HBSS) was prepared with sterile distilled water.
2.2. Biosynthesis of Ag-NPs. TSB medium was prepared, sterilized, and inoculated with a fresh growth of $P$. aeruginosa ATCC 27853, being incubated at $37^{\circ} \mathrm{C}$ for $24 \mathrm{~h}$. After the incubation time, the culture was centrifuged at $10,000 \mathrm{rpm}$ and the culture supernatant was used for the synthesis of Ag-NPs. Different concentrations of $P$. aeruginosa culture supernatant $(10,30$, and $50 \%$ by volume) were separately added to the reaction vessels containing silver nitrate at different concentrations $(1,5$, and $10 \mathrm{mM})$.

2.3. Characterization of Ag-NPs. The bioreduction of the $\mathrm{Ag}^{+}$ions was monitored at regular intervals by sampling aliquots $(2 \mathrm{~mL})$ of the reaction mixture and measuring the UV-vis spectrum of the mixture. UV-vis spectra of these samples aliquots were recorded from 200 to $800 \mathrm{~nm}$ on a Shimadzu UV-vis spectrophotometer at room temperature. The colloidal stability of Ag-NPs was evaluated by zeta potential measurements using a Delsa ${ }^{\mathrm{TM}}$ Nano $\mathrm{C}$ instrument (Beckman Coulter). Furthermore, the biosynthesized nanoparticles were characterized using transmission electron microscopy (TEM). Morphological analysis of Ag-NPs was carried out using TEM images acquired with a JEM-JEOL 1120 EXII model microscope operating at $80 \mathrm{kV}$. Samples were prepared by adding one drop of the reaction mixture onto a holey carbon-coated copper TEM grid and allowing it to dry in air. The crystal structure and chemical composition of Ag-NPs were determined by X-ray diffraction (XRD) analysis using an X-ray diffractometer (PANalytical X-Pert Pro) with $\mathrm{Cu}$ K-alpha radiation that was operated at $40 \mathrm{kV}$ and $40 \mathrm{~mA}$ at $2 \theta$ range of $30-70^{\circ}$.

2.4. Bacterial Strains. The antimicrobial activity of biosynthesized Ag-NPs was examined in several representative Gram-positive and Gram-negative bacterial strains. The following Gram-positive microorganisms were evaluated: $S$. aureus ATCC 29213, methicillin-sensitive S. aureus (MSSA) clinical strain 1, MSSA clinical strain 2, MSSA clinical strain 3, methicillin-resistant S. aureus (MRSA), S. epidermidis ATCC 12228, and E. faecalis ATCC 29212. Among Gram-negative microorganisms were tested $P$. mirabilis clinical strain, $A$. baumannii clinical strain, E. coli ATCC 25922, E. coli clinical strain 1, E. coli clinical strain 2, P. aeruginosa ATCC 27853, and $K$. pneumoniae ATCC 700603. All bacterial strains were grown aerobically in $\mathrm{MHB}$ for $24 \mathrm{~h}$ at $37^{\circ} \mathrm{C}$.

2.5. Determination of Minimum Inhibitory Concentration and Minimum Bactericidal Concentration of the Ag-NPs and TimeDeath Assays. The standard tube dilution method on $\mathrm{MHB}$ was used to evaluate the antimicrobial efficacy of the AgNPs. Strains coming from cultures of $24 \mathrm{~h}$ in MHB medium were diluted to $10^{6} \mathrm{CFU} / \mathrm{mL}$ and incubated for $10 \mathrm{~min}$ at $37^{\circ} \mathrm{C}$. The Ag-NPs concentrations added to bacterial suspensions were ranged from 0.025 to $51.2 \mathrm{pM}$. Bacterial growth was observed at $18 \mathrm{~h}$ of incubation following the indications of the Clinical and Laboratory Standards Institute (CLSI). The lowest concentration of the Ag-NPs that inhibited bacterial growth was considered to be the minimum inhibitory concentration (MIC). Minimum bactericidal concentration 


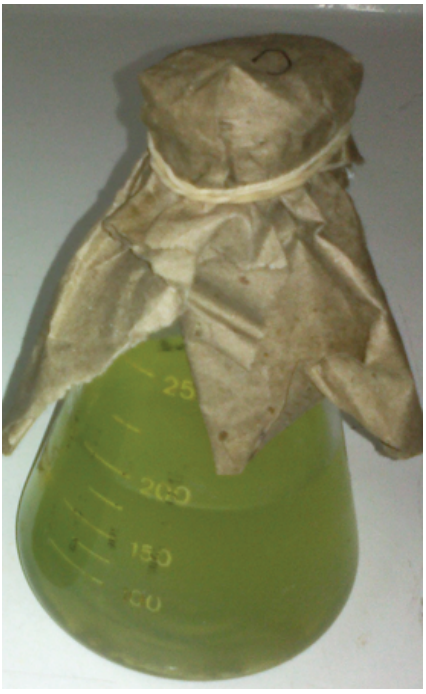

(a)

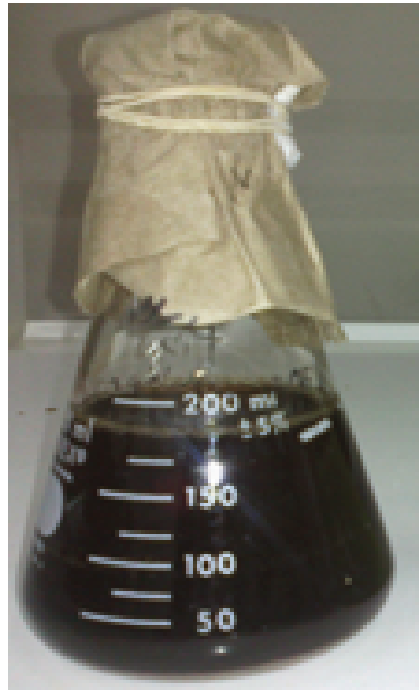

(b)

Figure 1: Biosynthesis of Ag-NPs. (a) Culture supernatant of P. aeruginosa without $\mathrm{Ag}^{+}$ions after $24 \mathrm{~h}$ of incubation. (b) Culture supernatant of $P$. aeruginosa with $\mathrm{AgNO}_{3} 10 \mathrm{mM}$ after $24 \mathrm{~h}$ of incubation.

(MBC) measured was the lowest concentration that reduced initial inoculums to $99.9 \%$. Time-death assays were conducted in the $S$. aureus and $E$. coli reference strains in the presence of $0.6 \mathrm{pM}$ Ag-NPs biosynthesized. Both strains at a starting inoculum of $10^{7} \mathrm{CFU} / \mathrm{mL}$ in $2 \mathrm{~mL}$ of $\mathrm{MHB}$ were incubated for $2.5 \mathrm{~h}$ at $37^{\circ} \mathrm{C}$ with constant agitation and then they were spiked with the nanoparticles. In different times, an aliquot of the bacterial suspension was collected, diluted in phosphate buffer solution, and plated on Mueller Hinton agar plates in the absence of Ag-NPs. Colonies were counted after 24 hours at $37^{\circ} \mathrm{C}$.

2.6. Neutrophils Preparation from Human Blood and Cell Viability Assay. Human neutrophils were isolated by a combined dextran/Ficoll-Hypaque sedimentation procedure. Sedimentation in dextran $6 \%$ was performed before gradient centrifugation. A mixture of Ficoll-Hypaque was then used to isolate the mononuclear cells from the remaining haematic cells. After sedimentation, hypotonic lysis of the erythrocytes was carried out. The neutrophil layer was washed twice and suspended in HBSS. Cell preparations were adjusted to $\sim 10^{6}$ cells $/ \mathrm{mL}$ for the assay.

The Trypan blue exclusion test was used to determine the number of viable cells present in a cell suspension exposed to Ag-NPs at $40 \mathrm{pM}$. In this test, a cell suspension is simply mixed with Trypan blue $0.02 \%$ and then examined to determine whether cells take up or exclude dye. In the protocol presented here, a viable cell will have a clear cytoplasm whereas a nonviable cell will show blue cytoplasm. Values of viability of treated cells were expressed as percentage of that from corresponding control cells.

2.7. Ethics Statement. Healthy volunteers were involved in this study for the human blood donation and all participants signed written informed consent before participation. This study was approved by the Chemical School Institutional Review Board and complies with the Argentinean (ANMAT 5330/97) and international (Declaration of Helsinki) principles and bioethical codes.

\section{Results and Discussion}

Addition of different concentrations of $P$. aeruginosa culture supernatant $(10,30$, and $50 \%$ by volume) to aqueous $\mathrm{AgNO}_{3}$ solution at different concentrations (1, 5, and $\left.10 \mathrm{mM}\right)$ resulted in the biosynthesis of Ag-NPs. However, the best compromise to generate higher amount of Ag-NPs with lower polydispersity was reached with a $10 \mathrm{mM} \mathrm{AgNO}_{3}$ solution and a $P$. aeruginosa culture supernatant concentration at $30 \%$ by volume. Figures $1(\mathrm{a})$ and 1 (b) display the visual change in color from greenish to yellowish brown of the culture supernatant incubated at $37^{\circ} \mathrm{C}$ with $\mathrm{Ag}^{+}$ions after $24 \mathrm{~h}$ of reaction, whereas no color change could be observed in culture supernatant without $\mathrm{AgNO}_{3}$. The bioreduction of the $\mathrm{Ag}^{+}$ions was confirmed by UV-vis spectroscopy as shown in Figure 2. Among the UV-vis spectra, a strong-broad absorption band centered at about $420 \mathrm{~nm}$ is observed and assigned to a surface plasmon [13], indicating the presence of Ag-NPs biosynthesized using the $P$. aeruginosa culture supernatant, while the absorption peak centered at around $300 \mathrm{~nm}$ is attributed to the silver ions. The zeta potential of Ag-NPs in the present study was found to be $-36.0 \mathrm{mV}$ suggesting that the repulsive forces between the nanoparticles would be responsible for electrostatic stability. This proves evidence that Ag-NPs were dispersed in the medium. Morphology and size distribution of Ag-NPs obtained were examined by transmission electron microscopy (TEM). A representative TEM image and a particle size histogram of the biosynthesized nanoparticles by extracellular matrix from $P$. aeruginosa are 
TABLE 1: Minimum inhibitory concentration (MIC) of Ag-NPs and ciprofloxacin and minimum bactericidal concentration (MBC) of Ag-NPs for different bacterial species.

\begin{tabular}{lccc}
\hline Bacterial strain & Ag-NPs & Ciprofloxacin \\
MIC $(\mu \mathrm{M})$
\end{tabular}

MSSA: methicillin-sensitive $S$. aureus; MRSA: methicillin-resistant S. aureus.

shown in Figures 3(a) and 3(b), respectively. It can be seen that the nanoparticles are spherical and roughly spherical and relatively uniform in diameter between 25 and $45 \mathrm{~nm}$. A possible mechanism that may explain the biosynthesis of Ag-NPs is considering that the NADH-dependent nitrate reductase, which is an enzyme secreted by $P$. aeruginosa, may be responsible for the reduction of $\mathrm{Ag}^{+}$to $\mathrm{Ag}^{0}$ and the subsequent Ag-NPs formation. The bioreduction may occur by means of the electrons from NADH where the NADHdependent reductase can act as a carrier [9, 11]. An X-ray diffraction pattern of the biosynthesized Ag-NPs is shown in Figure 4 . Three peaks at $38.1^{\circ}, 44.2^{\circ}$, and $64.5^{\circ}$ corresponding to the (111), (200), and (220) planes of silver were confirmed using standard powder diffraction data of JCPDS number 040783. All peaks corresponded to a face centered cubic (fcc) symmetry. In addition to these representative peaks of fcc silver nanocrystal, other peaks can be observed in Figure 4 suggesting the crystallization of a bioorganic phase on the surface of nanoparticles and Ag-NPs stabilization [14].

The continuous selection of bacteria that are resistant to a wide range of antibiotics has led to the resurgence in the research of novel unconventional sources of antibiotics. Accordingly, the antimicrobial properties of the biosynthesized Ag-NPs against representative Gram-positive and Gram-negative bacterial pathogens were explored in this work. We challenged clinical and reference strains of $S$. aureus, S. epidermidis, E. faecalis, P. mirabilis, A. baumannii, E. coli, $P$. aeruginosa, and $K$. pneumonia with different concentrations of Ag-NPs (from 0.1 to $51.2 \mathrm{pM}$ ) using the conventional tube macrodilution method to determine MIC and MBC of the Ag-NPs (see Table 1).

It can be observed in Table 1 that the biosynthesized AgNPs were effective against all the bacterial species studied

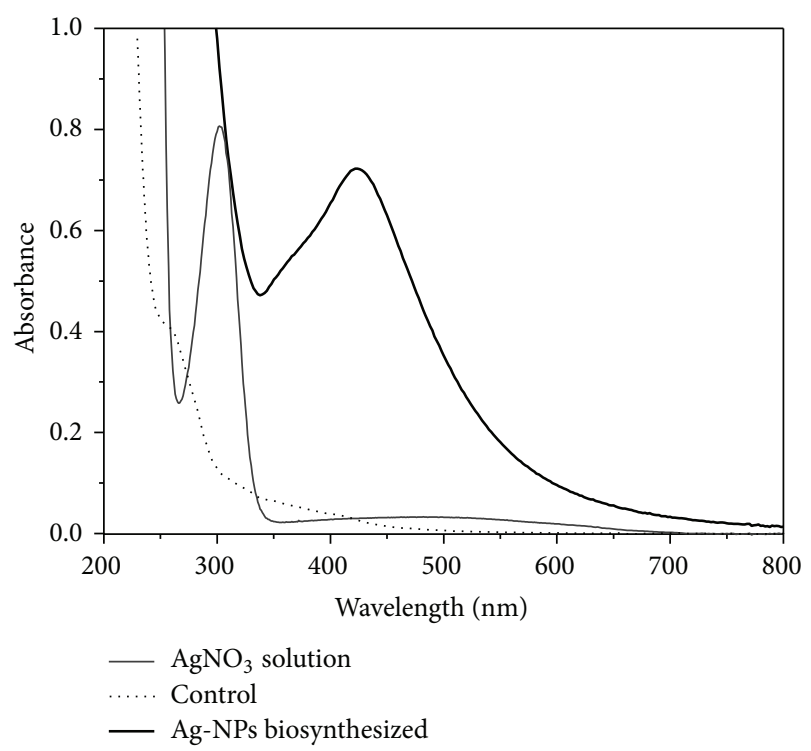

Figure 2: UV-visible spectra of Ag-NPs biosynthesized (black line), $\mathrm{AgNO}_{3}$ solution (gray line), and P. aeruginosa culture supernatant (control, dotted line). The absorption of Ag-NPs was recorded after the addition of a culture supernatant of $P$. aeruginosa at $30 \%$ by volume to $10 \mathrm{mM} \mathrm{AgNO}_{3}$ solution. The curve was recorded after $24 \mathrm{~h}$ of incubation.

and notable for their MIC at picomolar levels estimated between 0.4 and $6.4 \mathrm{pM}$. Comparing with a conventional clinical antibiotic, such as ciprofloxacin, the Ag-NPs obtained showed the higher growth inhibition effect against all of the tested bacterial species and significantly lower levels of concentration ( $\mu \mathrm{M}$ and $\mathrm{pM}$ for ciprofloxacin and Ag-NPs, resp.). 


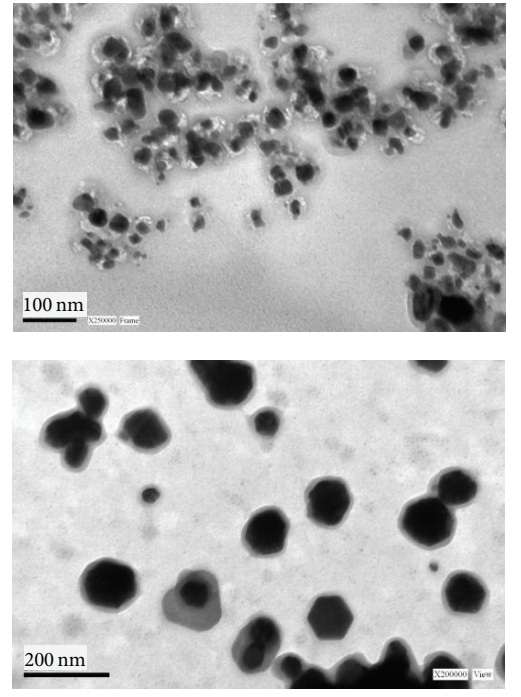

(a)

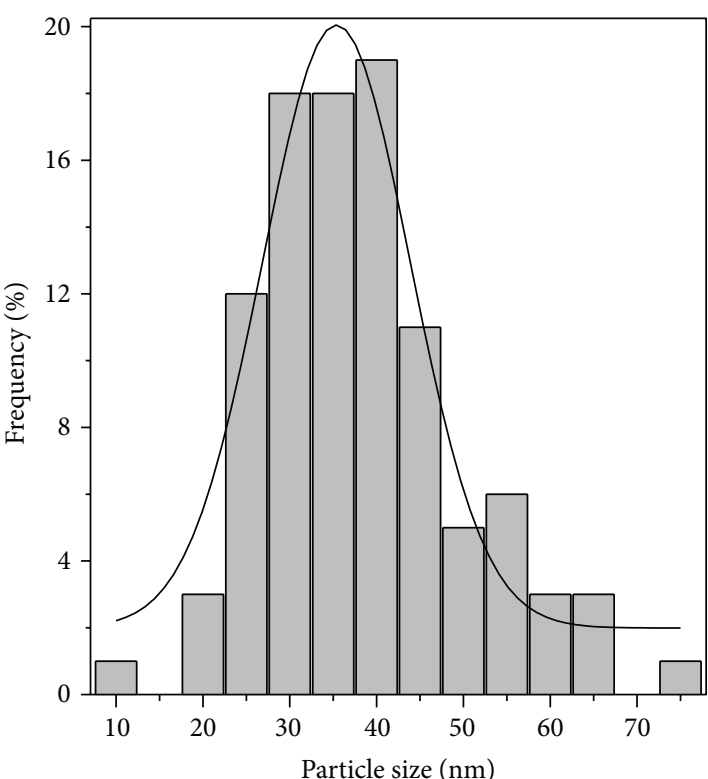

(b)

FIgURE 3: (a) Representative TEM images of Ag-NPs biosynthesized by reducing $\mathrm{Ag}^{+}$ions using a culture supernatant of $P$. aeruginosa. (b) Particle size histogram of Ag-NPs from TEM image showing the distribution of nanoparticles.

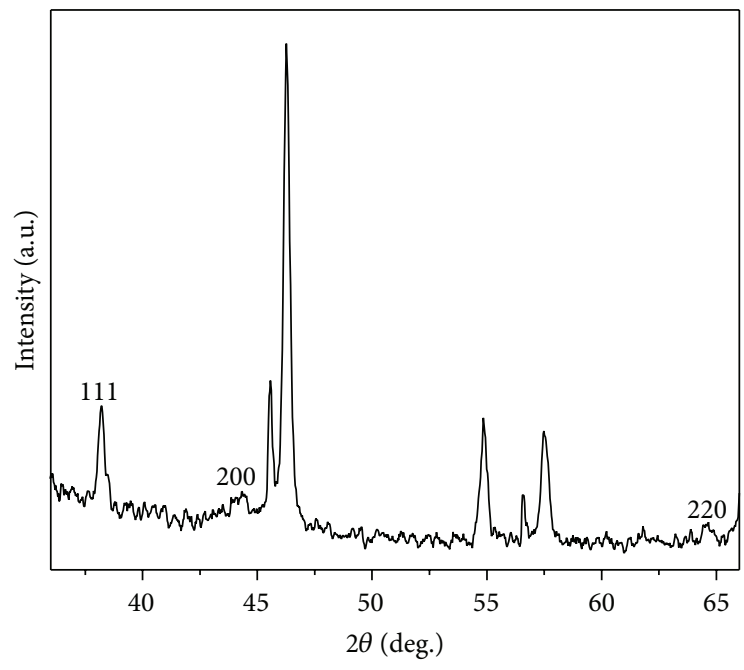

FIGURE 4: XRD spectrum of the biosynthesized Ag-NPs.

These results demonstrated that Ag-NPs may be used as potential antimicrobial agents and suggest the broad spectrum nature of their antimicrobial activity. The MIC values observed for $P$. aeruginosa and S. epidermidis were higher than for other bacterial strains, which could be explained for their capacity to form biofilm [15] and then to reduce the Ag-NPs-mediated antimicrobial action. Considering the $\mathrm{MBC} / \mathrm{MIC}$ ratio as a measure of the bactericidal power of an antimicrobial agent (bactericidal agent: $\mathrm{MBC} / \mathrm{MIC} \leq$ 2; bacteriostatic agent: $\mathrm{MBC} / \mathrm{MIC}>2$ ), the results listed in Table 1 allow pointing out a bactericidal activity of Ag-NPs in the bacterial species tested. Additionally, the

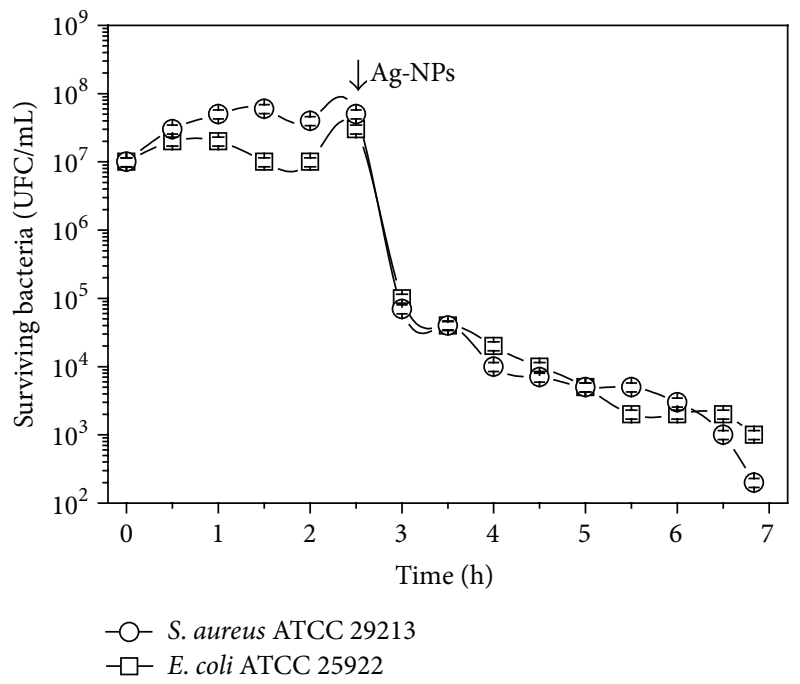

FIgUre 5: Time-death curves for S. aureus ATCC 29213 (-O-) and E. coli ATCC 25922 (-口-) using 0.6 pM Ag-NPs biosynthesized.

bactericidal kinetics of Ag-NPs biosynthesized were analyzed from time-death curve experiments using $S$. aureus ATCC 29213 and E. coli ATCC 25922, as models for Grampositive and Gram-negative bacteria, respectively. The results obtained showed a reduction of $3 \log _{10}$ after $4 \mathrm{~h}$ of incubation with an Ag-NPs concentration at $0.6 \mathrm{pM}$ (see Figure 5). Ag-NPs were powerful bactericidal agents against clinical pathogenic strains of methicillin-resistant S. aureus, A. baumannii, and E. coli, which have been considered some of the most virulent multidrug-resistant microorganisms for the human population [16]. This is a markedly promising 
result since the use of the biosynthesized Ag-NPs may be one of the approaches for overcoming bacterial resistance and playing an advanced key role in pharmacotherapeutics.

The mechanism of the Ag-NPs-mediated bactericidal effect remains to be understood. Several studies propose that Ag-NPs attach to the cell wall affecting its membrane integrity, thus disturbing permeability and respiration functions of the cell [9]. Likewise, the antibacterial activity of Ag-NPs is size dependent, and smaller Ag-NPs having the large surface area available for interaction are more effective antimicrobial agents than larger ones. Then, it is possible that Ag-NPs not only interact with the cell membrane, but can also penetrate inside the bacteria [8]. Another possible mechanism involved in the antimicrobial activity of Ag-NPs is the release of $\mathrm{Ag}^{+}$ions that play a partial but important role in their bactericidal effect [9].

Cell viability in response to Ag-NPs was estimated by Trypan blue exclusion test for cells in contact with much higher Ag-NPs concentrations than the MIC/MBC determined. After $30 \mathrm{~min}$ and $3 \mathrm{~h}$ incubation, the cell viability was greater than $80 \%$ and $50 \%$, respectively. These preliminary results demonstrated that the biosynthesized Ag-NPs have a negligible cytotoxic effect in human neutrophils even after $3 \mathrm{~h}$ of exposure to nanoparticles, suggesting low toxicity to the host. Thus, the unconventional antimicrobial agent obtained may be used in patients without side effect, being an alternative to control the infectious diseases caused by different pathogenic bacteria.

\section{Conclusion}

We reported a simple and green chemistry approach for the biological synthesis of Ag-NPs using the culture supernatant of a $P$. aeruginosa reference strain at $37^{\circ} \mathrm{C}$ and without any harmful reducing agents. The nanoparticles were characterized by means of UV-vis spectroscopy and transmission electron microscopy. TEM analysis confirmed the relatively uniform distribution of Ag-NPs and their roughly spherical shapes. The antimicrobial activity of the biosynthesized AgNPs was evaluated and it was found that this nanomaterial at picomolar concentration levels has bactericidal activity against representative human Gram-positive and Gramnegative pathogens including clinically isolated multidrugresistant bacteria such as methicillin-resistant $S$. aureus, $A$. baumannii, and E. coli. This is notable since Ag-NPs have proved to be effective antibacterial agents regardless of the drug-resistance mechanisms that exist in human pathogenic microorganisms and may be a potential candidate as effective broad spectrum bactericidal agents and nontoxic to the host.

\section{Competing Interests}

The authors declare no competing interests regarding the publication of this paper.

\section{Authors' Contributions}

Melisa A. Quinteros and Ivana M. Aiassa Martínez contributed equally to this work.

\section{Acknowledgments}

The authors wish to acknowledge the financial support of CONICET (PIP 11220130100702CO, PIO 14520140100013CO) and ANPCyT-FONCyT (PICT $2014 \mathrm{~N}^{\circ}$ 1663) from Argentina and SECyT-UNC from Córdoba, Argentina. Melisa A. Quinteros and Ivana M. Aiassa Martínez thank CONICET for the doctoral and postdoctoral fellowships, respectively. The authors would also like to thank Juan Carlos Fraire for assistance in TEM experiments.

\section{References}

[1] B. Spellberg, R. Guidos, D. Gilbert et al., "The epidemic of antibiotic-resistant infections: a call to action for the medical community from the infectious diseases society of America," Clinical Infectious Diseases, vol. 46, no. 2, pp. 155-164, 2008.

[2] D. J. Diekema, K. J. Dodgson, B. Sigurdardottir, and M. A. Pfaller, "Rapid detection of antimicrobial-resistant organism carriage: an unmet clinical need," Journal of Clinical Microbiology, vol. 42, no. 7, pp. 2879-2883, 2004.

[3] A. R. M. Coates and Y. Hu, "Novel approaches to developing new antibiotics for bacterial infections," British Journal of Pharmacology, vol. 152, no. 8, pp. 1147-1154, 2007.

[4] M. J. Hajipour, K. M. Fromm, A. Akbar Ashkarran et al., "Antibacterial properties of nanoparticles," Trends in Biotechnology, vol. 30, no. 10, pp. 499-511, 2012.

[5] M.-A. Neouze, "Nanoparticle assemblies: main synthesis pathways and brief overview on some important applications," Journal of Materials Science, vol. 48, no. 21, pp. 7321-7349, 2013.

[6] L. Zhang, F. X. Gu, J. M. Chan, A. Z. Wang, R. S. Langer, and O. C. Farokhzad, "Nanoparticles in medicine: therapeutic applications and developments," Clinical Pharmacology and Therapeutics, vol. 83, no. 5, pp. 761-769, 2008.

[7] H. M. C. De Azeredo, "Antimicrobial nanostructures in food packaging," Trends in Food Science and Technology, vol. 30, no. 1, pp. 56-69, 2013.

[8] M. Rai, A. Yadav, and A. Gade, "Silver nanoparticles as a new generation of antimicrobials," Biotechnology Advances, vol. 27, no. 1, pp. 76-83, 2009.

[9] S. Eckhardt, P. S. Brunetto, J. Gagnon, M. Priebe, B. Giese, and K. M. Fromm, "Nanobio silver: its interactions with peptides and bacteria, and its uses in medicine," Chemical Reviews, vol. 113, no. 7, pp. 4708-4754, 2013.

[10] K. N. Thakkar, S. S. Mhatre, and R. Y. Parikh, "Biological synthesis of metallic nanoparticles," Nanomedicine: Nanotechnology, Biology, and Medicine, vol. 6, no. 2, pp. 257-262, 2010.

[11] N. I. Hulkoti and T. C. Taranath, "Biosynthesis of nanoparticles using microbes-a review," Colloids and Surfaces B: Biointerfaces, vol. 121, pp. 474-483, 2014.

[12] C. G. Kumar and S. K. Mamidyala, "Extracellular synthesis of silver nanoparticles using culture supernatant of Pseudomonas aeruginosa," Colloids and Surfaces B: Biointerfaces, vol. 84, no. 2, pp. 462-466, 2011.

[13] P. Mulvaney, "Surface plasmon spectroscopy of nanosized metal particles," Langmuir, vol. 12, no. 3, pp. 788-800, 1996. 
[14] N. Basavegowda and Y. Rok Lee, "Synthesis of silver nanoparticles using Satsuma mandarin (Citrus unshiu) peel extract: a novel approach towards waste utilization," Materials Letters, vol. 109, pp. 31-33, 2013.

[15] K. Kalishwaralal, S. BarathManiKanth, S. R. K. Pandian, V. Deepak, and S. Gurunathan, "Silver nanoparticles impede the biofilm formation by Pseudomonas aeruginosa and Staphylococcus epidermidis," Colloids and Surfaces B: Biointerfaces, vol. 79, no. 2, pp. 340-344, 2010.

[16] A. L. Cohen, D. Calfee, S. K. Fridkin et al., "Recommendations for metrics for multidrug-resistant organisms in healthcare settings: SHEA/HICPAC position paper,' Infection Control and Hospital Epidemiology, vol. 29, no. 10, pp. 901-913, 2008. 

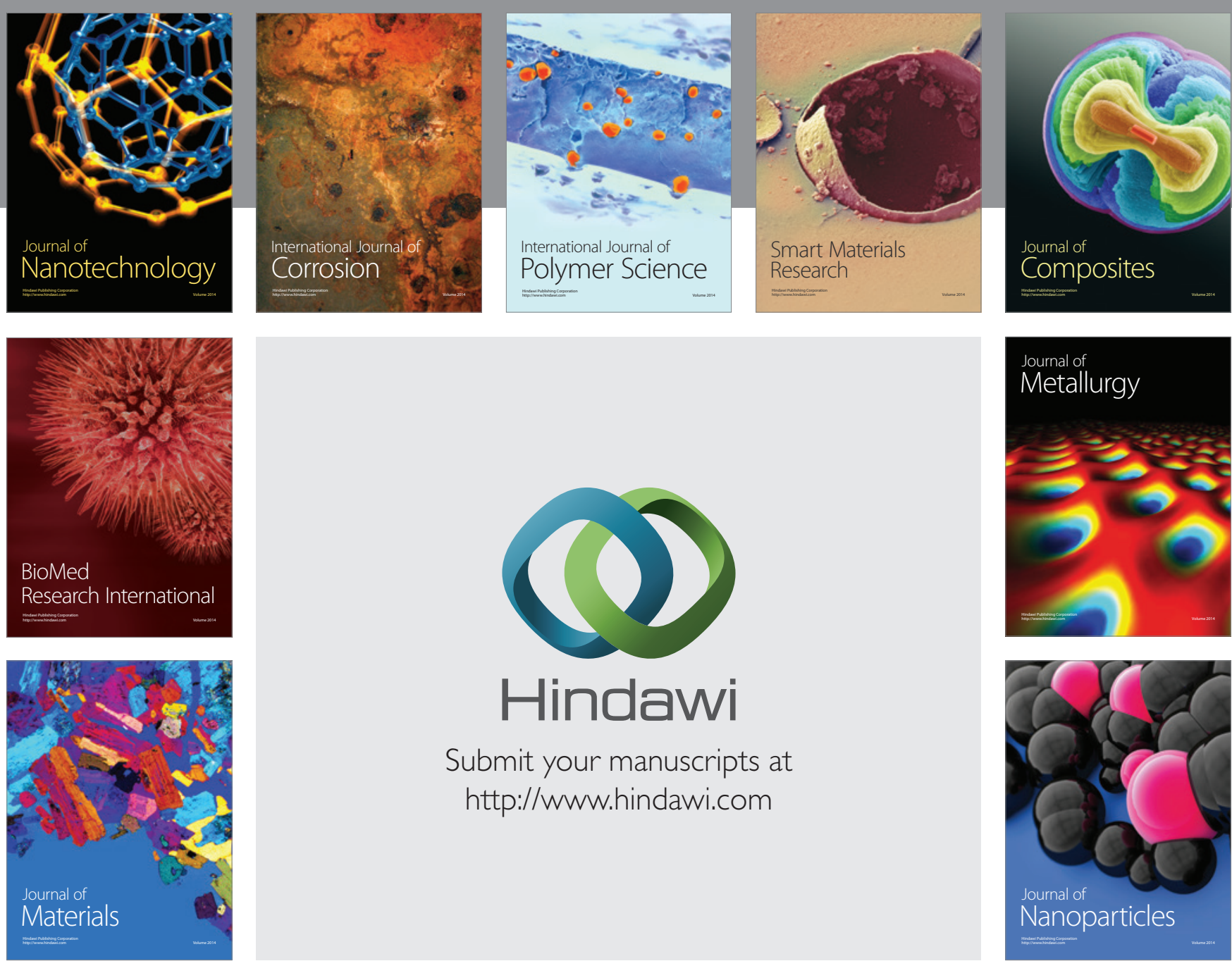

\section{Hindawi}

Submit your manuscripts at

http://www.hindawi.com

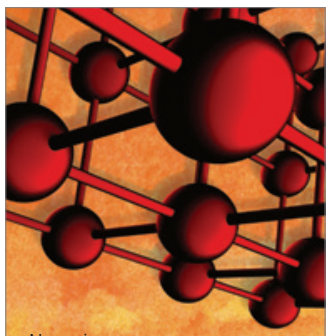

Materials Science and Engineering
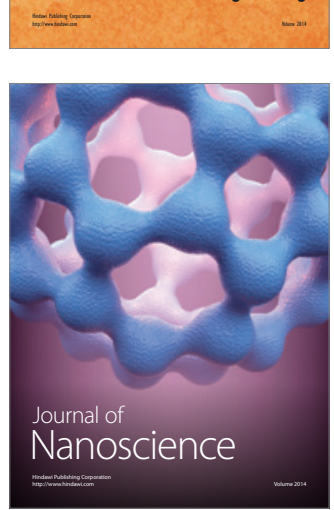
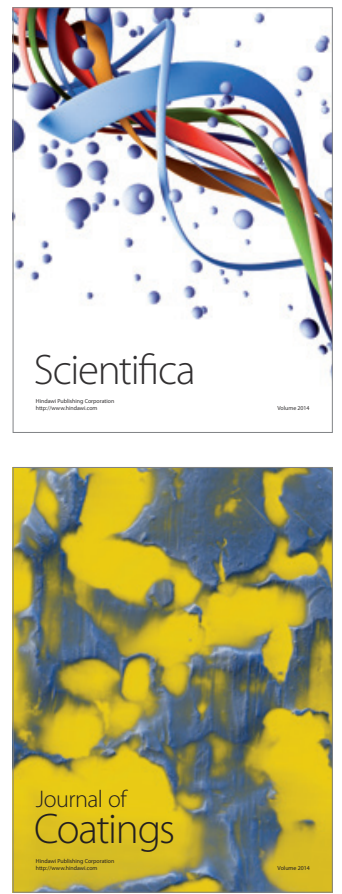
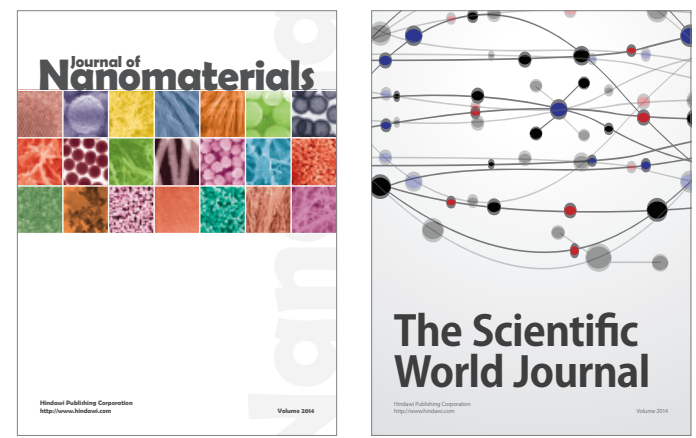

The Scientific World Journal
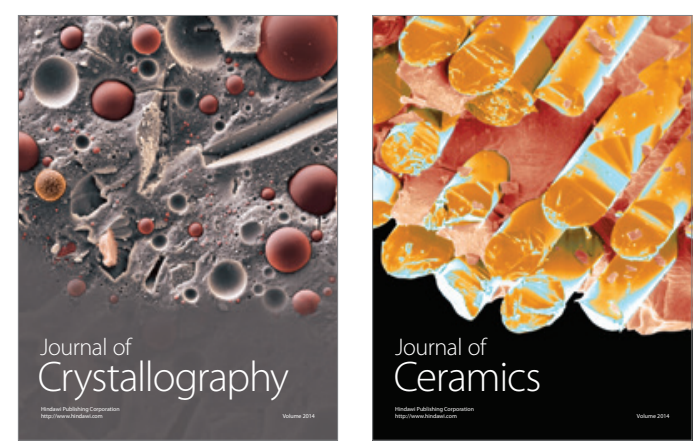
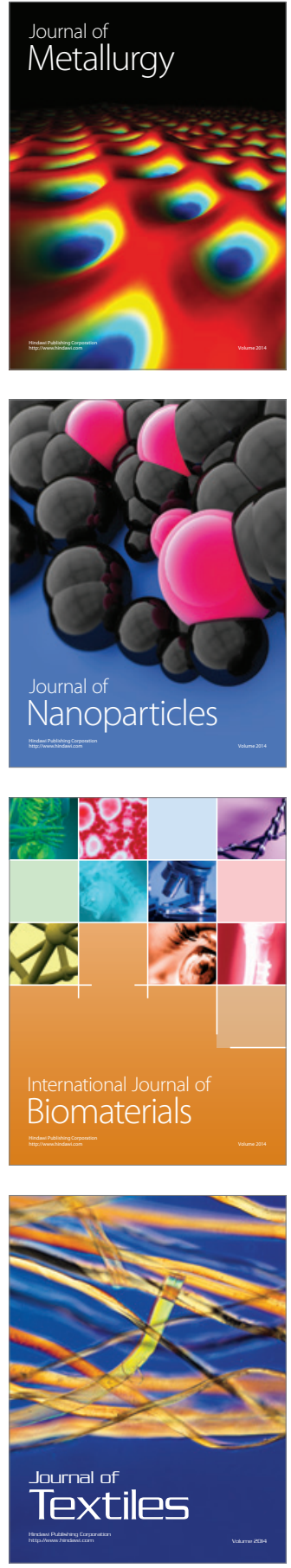